Artículo de investigación.

Cómo citar: A. Hernández, C. Velandia, y M. Herrera "Una revisión de las contribuciones de la dinámica de sistemas en la transición de vehículos eléctricos", Inventum, vol. 14, no. 27, pp. 89-102. doi: 10.26620/uniminuto.inventum.14.27.2019.89-102

Editorial: Corporación Universitaria Minuto de Dios - UNIMINUTO.

ISSN: $1909-2520$

eISSN: $2590-8219$

Fecha de recibido: mayo 01 de 2019

Fecha de aprobado: junio 30 de 2019

Fecha de publicación: septiembre 01 de 2019

Conflicto de intereses: los autores han declarado que no existen intereses en competencia.

\section{Una revisión de las contribuciones de la dinámica de sistemas en la transición de vehículos eléctricos}

\author{
A review of the contributions of system \\ dynamics on the electric vehicle \\ transition
}

\author{
Uma Revisão das Contribuições para a \\ Dinâmica de Sistemas na Transição de \\ Veículos Elétricos
}

\begin{abstract}
Resumen
La transición de tecnologías intensivas en el uso de combustibles fósiles a tecnologías limpias ha tomado relevancia en las últimas décadas. En este sentido, estrategias para la implementación de alternativas de transporte que mitiguen la emisión de dióxido de carbono han sido ampliamente estudiadas en la literatura. A pesar de las estrategias de transición desarrolladas, nuevos desafíos enfrentan la transición de tecnologías limpias (ejemplo, vehículos eléctricos y energías renovables) que requieren de una intervención sistémica. La dinámica de sistemas ha sido por excelencia una metodología apropiada para el análisis de transición de vehículos eléctricos. Este artículo realiza una revisión que contribuye con las oportunidades y desafíos planteados desde la literatura para enfrentar la transición tecnológica en el sector transporte, específicamente los vehículos eléctricos.
\end{abstract}

Palabras clave: transición tecnológica, simulación, vehículos eléctricos, revisión de literatura, dinámica de sistemas.

\begin{abstract}
The transition of fossil fuel technology to clean technologies has taken relevance in the last decade. In this sense, strategies for the implementation of alternatives transport that mitigate $\mathrm{CO}_{2}$ emissions have been thoroughly studied in the literature. Despite the strategies of transitions developed, new challenges face the transition of clean technologies (e.g., electric vehicle and renewable energy) which require a systemic intervention. System dynamics has been a useful methodology to analyse the electric vehicles transition. This paper fulfils a revision that contributes to the challenges and opportunities from literature to face the technological transition in the transport sector, specifically electric vehicles.
\end{abstract}

Keywords: Technological transition, simulation, electric vehicles, literature review, system dynamics. 


\section{Resumo}

A transição de tecnologias com utilização intensiva de combustíveis fósseis para tecnologias limpas tornou-se relevante nas últimas décadas. Nesse sentido, estratégias para a implementação de alternativas de transporte que mitiguem a emissão de dióxido de carbono têm sido amplamente estudadas na literatura. Apesar das estratégias de transição desenvolvidas, novos desafios surgem a transição para tecnologias limpas (por exemplo, veículos elétricos e energias renováveis) que requerem intervenção sistêmica. A dinâmica de sistemas tem sido, por excelência, uma metodologia adequada para a análise de transição de veículos elétricos. Este artigo fornece uma revisão que contribui para as oportunidades e desafios colocados pela literatura para abordar a transição tecnológica no setor de transportes, especificamente veículos elétricos.

Palavras-chave: transição tecnológica, simulação, veículos elétricos, revisão da literatura, dinâmica de sistemas. 


\section{INTRODUCCIÓN}

El incremento de las emisión de $\mathrm{CO}_{2}$ a nivel mundial ha llevado a los Gobiernos de diferentes países a tomar medidas para mitigar el problema [1], [2]. Una gran parte de las emisiones es producida por el sector transporte, lo que causa efectos sobre la salud y el ambiente [3]. La falta de políticas adecuadas para el desarrollo de infraestructura de transporte también ha generado problemas de congestión que intensifican el problema del nivel de emisiones producidas por este sector [4], [5]. A pesar que estas preocupaciones han sido abordadas desde la literatura utilizando diferentes métodos, tales como la optimización [6], la simulación también ha sido empleada frecuentemente para analizar estos problemas [7], [8].

La transición de vehículos eléctricos es un tema de gran interés para los decisores de política. Entender las dinámicas de transición tecnológica pueden contribuir a mejorar el bienestar de una población. Estudios previos se han enfocado en entender la política y la dinámica de transición de los vehículos eléctricos [2], [9], [10]. Este artículo presenta una revisión de literatura enfocada a entender las siguientes preguntas: ¿cómo ha sido la evolución de la literatura del modelado de política para la transición de vehículos eléctricos? ¿Cuáles son las tendencias y desafíos del modelamiento de la transición de vehículos eléctricos con dinámica de sistemas?

A medida que incrementa la necesidad de transporte en una ciudad o región, la interacción entre los actores y las decisiones en términos de transporte cobran mayor complejidad [11]. En este sentido, la simulación ha sido ampliamente utilizada para detallar problemas que emergen de las relaciones complejas entre las decisiones y actores involucrados en el sistema. El modelado mediante dinámica de sistemas ha cobrado suma importancia para analizar los sistemas de transporte y las complejidades que ello implica [7].

La dinámica de sistemas es una metodología de simulación desarrollada por Jay Forrester a mediados de los años 50. Esta metodología ha sido empleada extensamente para abordar problemas de complejidad social, económica y ambiental [12], [13]. Sus primeros trabajos fueron enfocados a entender la dinámica de la industria y de allí se ha ampliado su rango a otros problemas de orden mundial.

La simulación con un enfoque de dinámica de sistemas representa los comportamientos de un sistema a través de la construcción de una plataforma que relaciona variables mediante un sistema de ecuaciones diferenciales [14]. Esto permite la representación del comportamiento de dicho sistema en el tiempo y los efectos e implicaciones a largo plazo de las variables en estudio. En el caso de transporte, la simulación mediante este enfoque ha tomado gran relevancia debido a las variables que representa este medio y que generan relaciones complejas de análisis en la toma de decisión.

Este artículo presenta una revisión de la literatura de los trabajos más relevantes en la transición de vehículos eléctricos, en los cuales se ha hecho uso de la dinámica de sistemas como herramienta de modelación. Adicionalmente, se proponen algunas categorías de análisis para entender las relaciones y contribuciones de la literatura.

Las siguientes secciones que conforman este artículo se encuentran divididas como se explica. La sección 2 presenta los conceptos básicos de la representación de transición utilizados por la dinámica de sistemas. La sección 3 exhibe la metodología empleada en la revisión de literatura. La sección 4 muestra los resultados obtenidos de la revisión, las tendencias y desafíos que abordan los estudios de transición de vehículos eléctricos. La sección 5 presenta las conclusiones de la revisión realizada.

\section{CONCEPTOS PRELIMINARES DE MODELADO CON DINÁMICA DE SISTEMAS}

A pesar de que se utilizan diferentes métodos para modelar la transición de vehículos eléctricos en una región [15], [16], la dinámica de sistemas ha permitido entender a partir de una intervención sistémica los retardos que afectan las transiciones tecnológicas [17]. La dinámica de sistemas contempla varios pasos que permiten entender y modelar las implicaciones de los retardos en las decisiones. Estos pasos contemplan [8]:

- Definición del problema.

- Construcción de modelo conceptual, a través de un diagrama causal.

- Construcción de un modelo de simulación soportado en un diagrama de flujos y niveles.

- Diseño de escenarios de simulación para entender las implicaciones de las políticas. 
La definición del problema contempla la identificación de los elementos y actores que intervienen en el sistema. Determinar el problema se convierte en el insumo de la construcción del modelo conceptual, lo cual implica conocer los elementos que interactúan (partes interesadas) y las relaciones y variables que influyen la toma de decisiones de las partes interesadas.

La construcción de un modelo conceptual conlleva un análisis profundo y sistémico que determine la hipótesis que explica el comportamiento dinámico de las variables. Por lo tanto, la representación del comportamiento dinámico puede estar mediada por un diagrama con ciclos causales que representan los efectos en las variables en las que confluyen las decisiones o políticas.

El modelo de simulación es representado como un diagrama de flujos y niveles, correlacionado con la hipótesis del comportamiento dinámico y que representa las variables del sistema. Este diagrama contiene un conjunto de ecuaciones diferenciales que serán útiles para entender los cambios de comportamiento en el tiempo.

Los modelos de simulación se caracterizan por realizar un análisis que responde a la pregunta: “¿qué pasa si...?”. Este análisis permite recrear escenarios de situaciones que pueden pasar en el largo plazo, los cuales evalúan la hipótesis del comportamiento dinámico.

Finalmente, un análisis de las relaciones sistémicas que existe entre las variables de un sistema permite construir escenarios para comprender los cambios de decisión y el diseño de una política. En el caso de las transiciones la dinámica de sistemas se convierte en un enfoque apropiado que contribuye a la intervención sistémica de las decisiones de transición a las tecnologías limpias [18] - [20]. Por supuesto, los modelos de simulación incluyen diferentes técnicas que permiten su valoración y pertinencia en diferentes aspectos: estructural, dimensional y comportamental.

\section{METODOLOGÍA DESARROLLADA}

Este artículo presenta una revisión de la literatura de las contribuciones de la dinámica de sistemas en la transición de vehículos eléctricos. Está revisión se realizó en dos fases: i) una revisión extensa en las contribuciones de la dinámica de sistemas en transporte y ii) una revisión detallada de las contribuciones de la dinámica de sistemas en el contexto de los vehículos eléctricos. En ambas fases se consideró un periodo de revisión entre 2012 y 2019 , excluyendo artículos publicados en memorias (publicaciones seriadas), esto debido a que el objetivo de las mismas es de difusión del conocimiento o divulgación. La revisión de literatura fue soportada en las bases de datos de Web of Sciences y Scopus, que fueron consideradas debido a su alto margen de calidad de revisión e indexación. Además, se tuvieron en cuenta para cada fase de revisión los descriptores: i) transport and system dynamics en la primera fase y ii) system dynamics and electric vehicles en la segunda fase. La prevalencia en la búsqueda de literatura tuvo en cuenta el número de citaciones obtenidos por publicación, utilizando el programa Publish or Perish. En este sentido, una breve descripción de las fases de la metodología empleada se muestra a continuación:

a) En la primera fase se identificaron 32 artículos que emplean la simulación con dinámica de sistemas aplicada a problemas de transporte. Además, se identificaron 5 tesis de maestría y de doctorado que abordan este problema. La revisión empleada tomó en cuenta aspectos fundamentales del artículo, tales como: título, resumen, palabras clave, metodología, resultados y conclusiones, por lo cual se descartaron otros enfoques propuestos por los autores en temas de transporte. Se destaca que en esta fase se incluyó una edición especial publicada en la revista System Dynamics Review.

b) En la segunda fase, se identificaron 25 artículos que abordan problemas de la expansión de vehículos eléctricos, soportados con la metodología de simulación de dinámica de sistemas. En estos artículos se destaca el número de citaciones de acuerdo a los temas que aborda la publicación (enfoques). Un análisis detallado que incluye las estrategias propuestas desde la simulación también fue considerado.

Las limitaciones de la metodología empleada contemplan los siguientes aspectos:

- No se toman en cuenta publicaciones anteriores a 2012, debido a que la revisión de literatura realizada busca las contribuciones y tendencias que ha tenido y puede tener la metodología de simulación con dinámica de sistemas en el análisis de la transición de vehículos eléctricos en varios países. 
- No se consideran otros enfoques de simulación, tales como simulación con agentes y simulación discreta, debido a que el estudio busca las aplicaciones de carácter estratégico en las cuales contribuye la dinámica de sistemas en los problemas de transición a vehículos eléctricos.

- Se limitan las bases de datos y no se incluyen otras bases de datos que podrían ser relevantes, para darle mayor importancia al nivel de impacto (es decir, número de citaciones) de las revistas indexadas en las bases de datos utilizadas.

- Un número considerable de publicaciones en el evento de la comunidad de dinámica de sistemas se excluyó, debido a que estos trabajos pueden ser investigaciones no concluidas.

\section{RESULTADOS OBTENIDOS}

Esta sección presenta los resultados obtenidos de la revisión de literatura. A partir de la revisión sistemática, se identificaron temáticas y conjunciones, que permitieron entender los desafíos y contribuciones de estudios previos que utilizan la simulación con dinámica de sistemas aplicada en el sector transporte.

\section{A. Evolución de la literatura}

La literatura en el análisis de políticas de transición de tecnologías limpias en transporte, soportadas a través de la dinámica de sistemas ha sido abundante [7], [21]. La metodología de simulación con dinámica de sistemas ha sido empleada para abordar varios aspectos enmarcados en los sistemas de transporte, tales como: políticas de mantenimiento y sus implicaciones en los sistemas de transporte público de pasajeros [22], [23]; mantenimiento de autopistas [24]; uso de la tierra y sus implicaciones en el sistema de transporte [25], [26]; análisis de polución urbana de los sistemas de transporte [2], [27]; evaluación de la inversión de infraestructura de transporte a nivel regional [28]; implicaciones económicas y ambientales en la gestión de transporte, particularmente de taxis [29], y diseño de política para el transporte urbano en regiones particulares de alta densidad poblacional [30]. Por lo tanto, la dinámica de sistemas ha mostrado ser una herramienta útil para abordar las interacciones de los sistemas de transporte. Si bien los trabajos publicados recientemente evalúan los impactos y reducción de las emisiones en el sistema de transporte [31]-[33], la transición y difusión de los vehículos eléctricos se ha convertido en un tema de gran interés en la comunidad científica [34], [35].

La figura 1 representa el grado de relevancia de las palabras clave utilizadas en las publicaciones consultadas. Este grado de relevancia asociado al crecimiento de publicaciones en la temática de vehículos eléctricos presenta diferentes tendencias, tales como: estudios enfocados en el análisis de la capacidad tecnológica (baterías), vehículos híbridos, diseño de política pública para la inserción de vehículos eléctricos y control y reducción de emisiones a partir de la adopción de vehículos eléctricos.

Figura 1. Relevancia de palabras claves empleadas

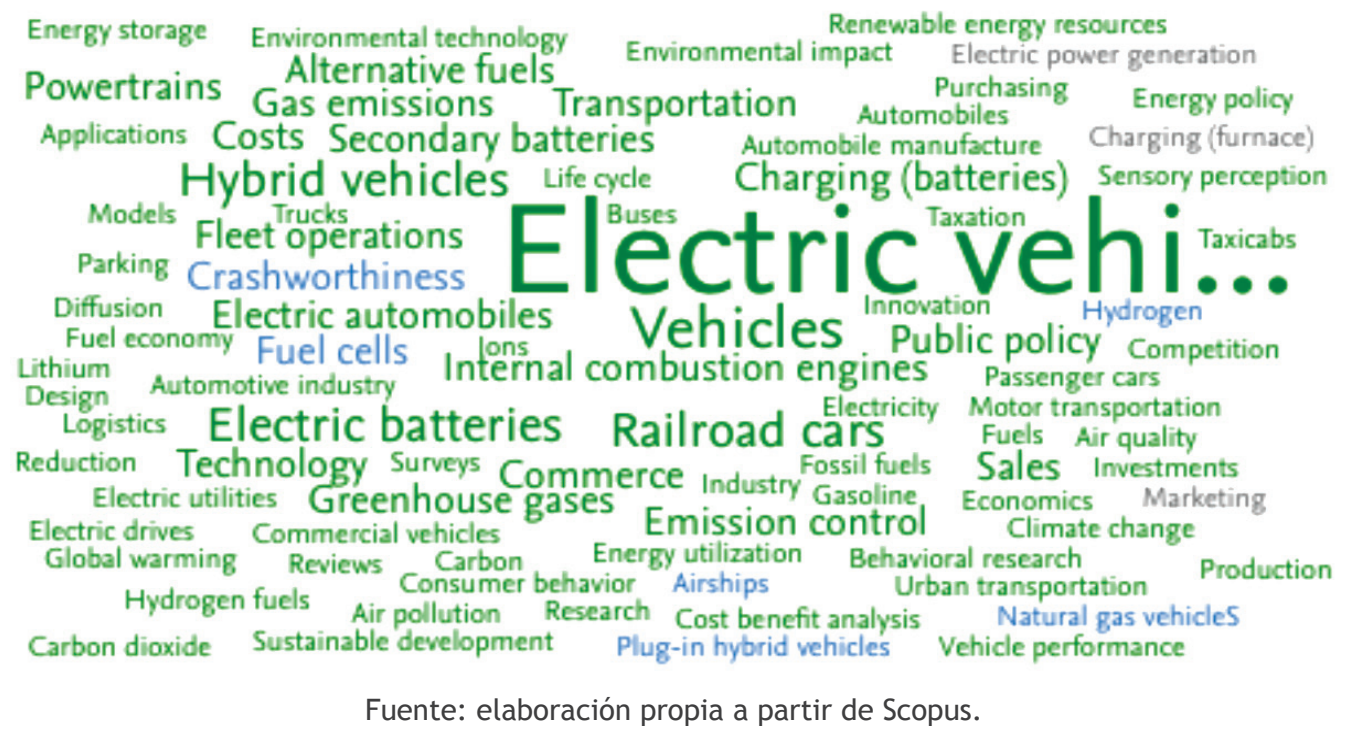


La figura 2 muestra la evolución de las publicaciones entre el periodo 2012 a 2019. Estas publicaciones muestran un interés particular en la implementación de tecnologías limpias para el sector de transporte. En el caso de las publicaciones realizadas en el año 2019, se identifica que la dinámica de sistemas ha sido empleada particularmente en el análisis de política para la adopción de vehículos eléctricos, su impacto en la reducción de emisiones y las implicaciones en la aceptación de los consumidores en el mercado automotriz. Se identifica que las publicaciones realizadas para el año 2012 abordaron adicionalmente problemas relacionados con la infraestructura que soporta la adopción de vehículos eléctricos y que también incluían el problema de reducción de emisiones.

Figura 2. Evolución de las publicaciones que abordan el tema de

transición de vehículos eléctricos soportados con dinámica de sistemas

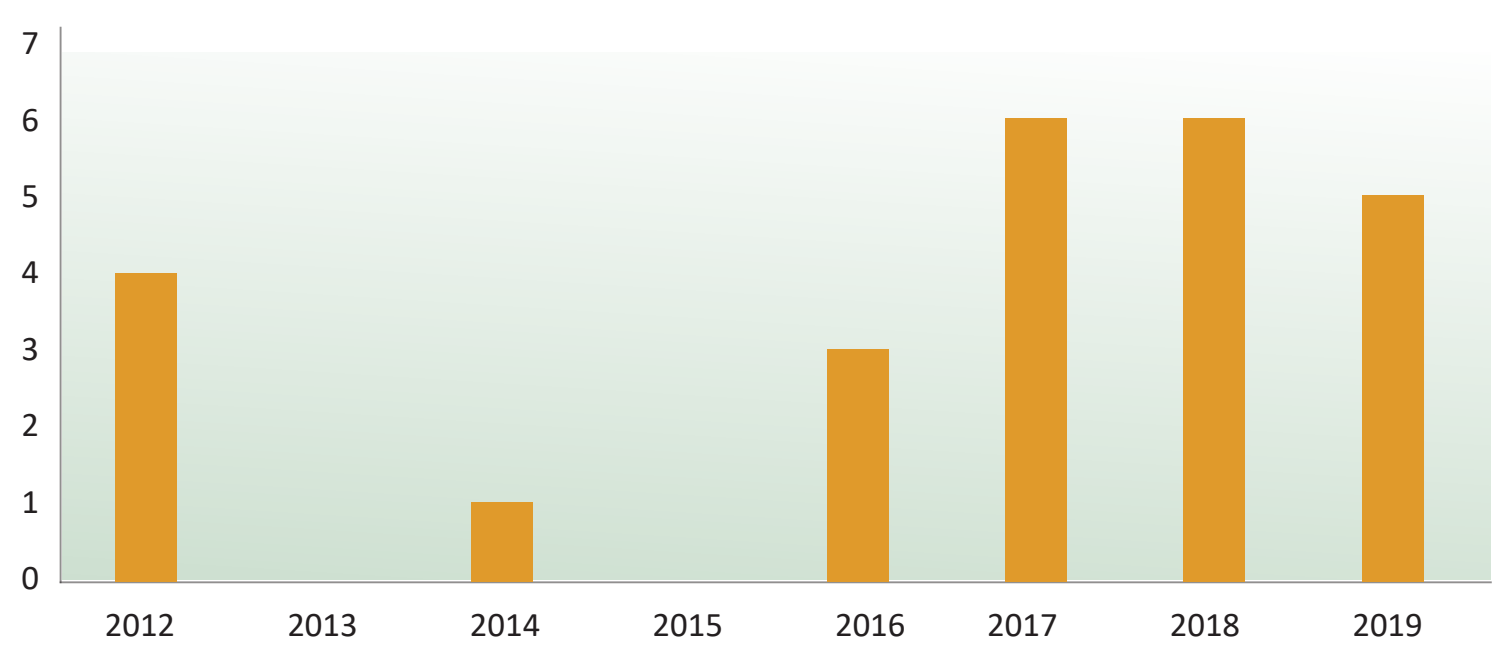

Años

Número de artículos

Fuente: elaboración propia a partir de Web of Sciences y Scopus.

A partir de la revisión de literatura se identificaron los autores con mayor citación de acuerdo a los descriptores system dynamics and electric vehicles, como se muestra en la figura 3 , y el apéndice I. Los autores con mayor número de citaciones convergen en abordar el tema relacionado con la reducción de emisiones con la implementación de políticas de adopción para vehículos eléctricos [7], [36]-[38]. Además, una perspectiva de la capacidad tecnológica relacionada con las baterías y autonomía de los vehículos eléctricos es modelada para evaluar los costos de estaciones de carga y baterías instaladas en los vehículos [39]. 
Figura 3. Autores con mayor número de citaciones

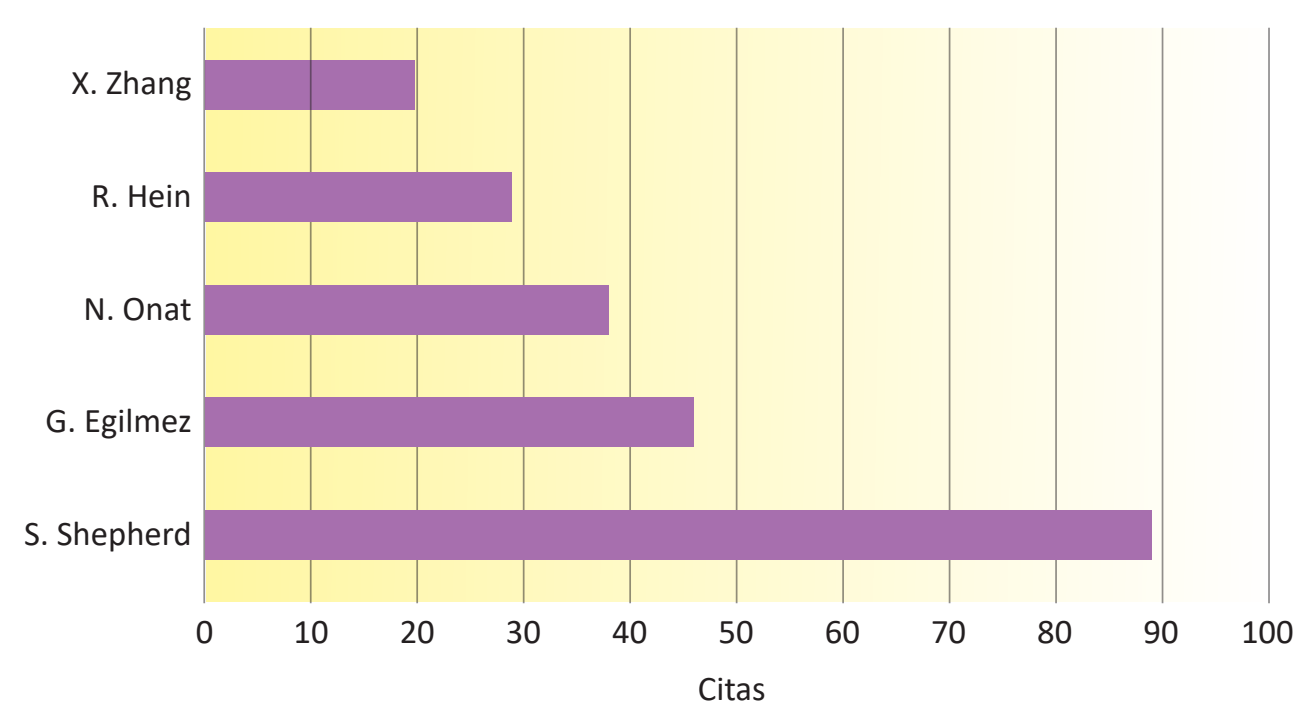

Fuente: elaboración propia a partir de Publish and Perish.

Con los resultados obtenidos a partir de la revisión de literatura, se logran identificar diferentes enfoques que han adoptado los autores para abordar la transición de vehículos eléctricos. La figura 4 muestra la relación de los términos relacionados entre los autores.

Por lo tanto, los artículos encontrados han sido clasificados en las siguientes categorías de análisis propuestas:

a) Evaluación de políticas para la adopción de vehículos eléctricos. b) Reducción de emisiones a partir de la transición de vehículos eléctricos.

c) Desarrollo de infraestructura para la transición de vehículos eléctricos.

d) Comportamiento del consumidor para la adopción de vehículos eléctricos.

e) Evaluación de políticas para la industria de vehículos eléctricos

f) Capacidad tecnológica de los vehículos eléctricos relacionada con las baterías.

Figura 4. Relaciones coincidentes entre los artículos seleccionados obtenidas a través de VOSviewer

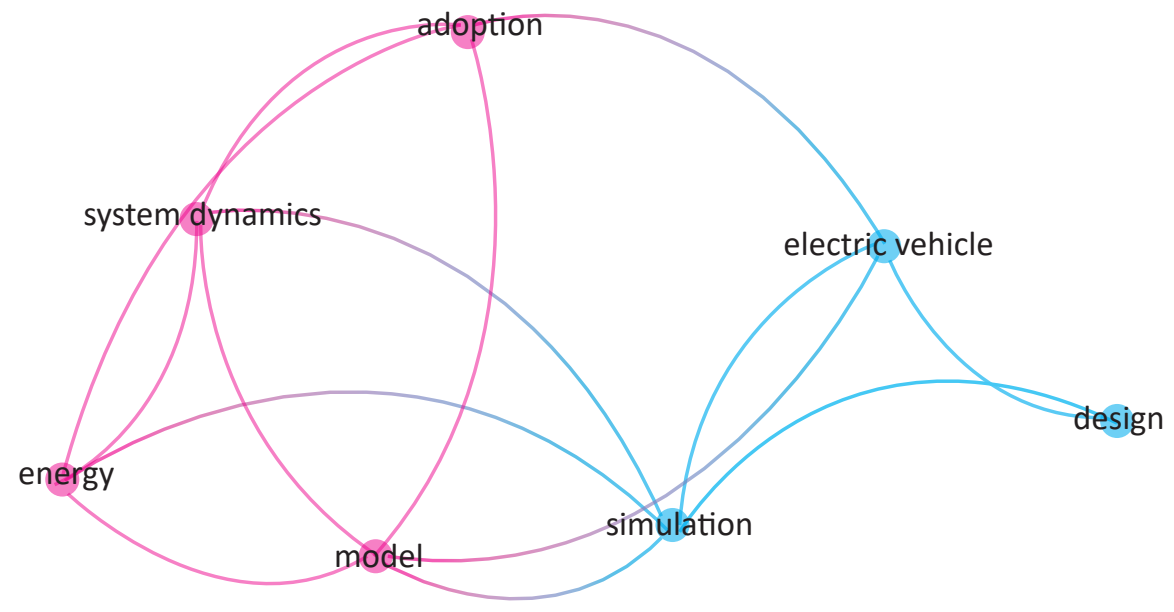

Fuente: elaboración propia. 
La figura 5 representa el conjunto del número de artículos que se han publicado en las categorías de análisis (a), (b) y (c). Es decir, en el caso de la adopción (a), emisiones (b) e infraestructura (c) se han publicado 16,7 y 5 artículos, respectivamente. De esta cantidad de artículos se puede identificar que 5 de ellos mezclan los aspectos (a) y (b). Mientras que únicamente 2 combinan los aspectos de emisiones e infraestructura. La evaluación de la adopción de vehículos eléctricos y la reducción de emisiones propone estrategias de mercado relacionadas con regímenes de subsidios para la transición de vehículos eléctricos [7], [40]. Estas estrategias también están acompañadas por la evaluación de los impactos sociales [37]. Además, se identifica el diseño de políticas que contemplan la categoría de análisis de infraestructura para el desarrollo de los vehículos eléctricos, estos trabajos formulan estrategias que combinan la asociación de instituciones públicas y privadas, como también los conflictos sociales que pueden incidir en la adopción e infraestructura necesaria [38], [41].

En este contexto de categorías, se identifica que no existen trabajos que combinen las categorías de emisiones, adopción e infraestructura.

Figura 5. Número de artículos publicados en las categorías de adopción, emisiones e infraestructura adoptados por los autores

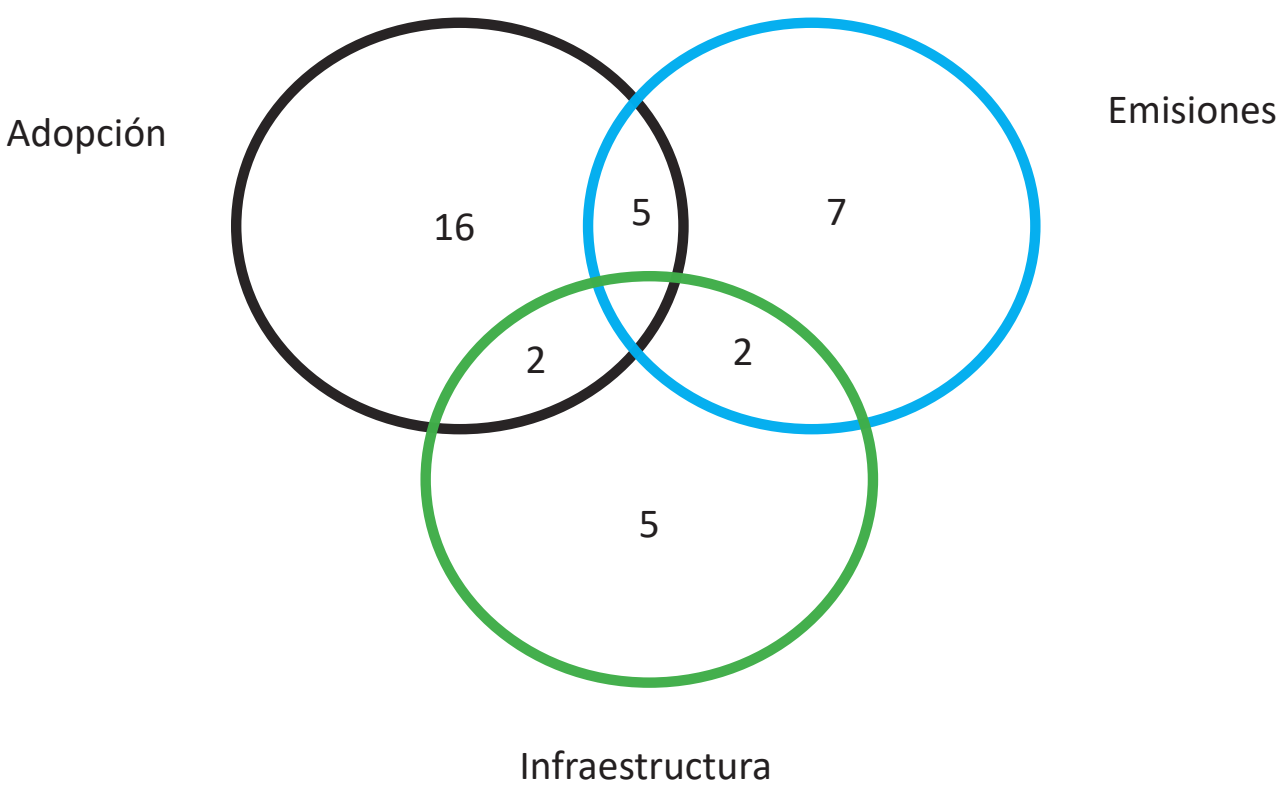

Fuente: elaboración propia.

Los aspectos de adopción de tecnología vehicular eléctrica también son abordados en la literatura con categorías que asocian la industria automotriz y el comportamiento del consumidor. En este último aspecto se ha simulado la influencia del reemplazo de vehículos y los incentivos al consumidor como elementos fundamentales de la adopción [42], [43]. Además, la adopción de tecnologías limpias requiere un análisis desde la oferta, en este caso, la industria automotriz, el cual es abordado por [44]. La figura 6 representa el número de artículos en estas categorías de análisis y su conjunción entre ellos. Se destaca que en este caso no existen trabajos que combinen la categoría de industria, adopción y comportamiento del consumidor. 
Figura 6. Número de artículos publicados en las categorías de adopción, industria y comportamiento del consumidor adoptados por los autores.

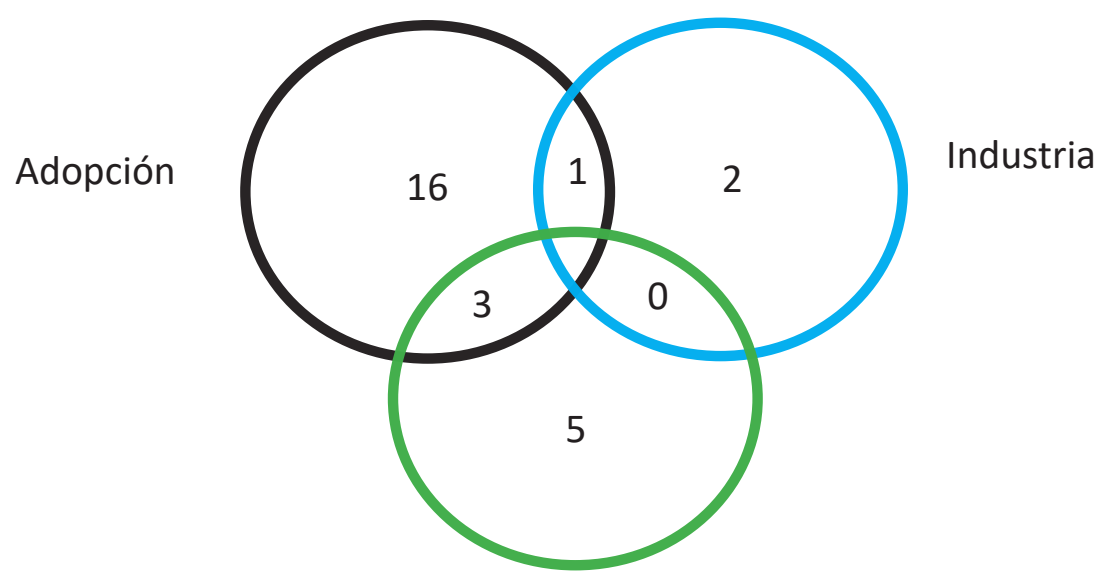

Comportamiento del consumidor

Fuente: elaboración propia.

La figura 7 presenta la conjunción entre las categorías de análisis de adopción, capacidad tecnológica (baterías) y comportamiento del consumidor. En este análisis se identifica que la categoría de comportamiento del consumidor se encuentra vinculada a la capacidad tecnológica de las baterías en los vehículos eléctricos [45]. En este caso particular la dinámica de sistemas ha sido levemente empleada en comparación con otras categorías de análisis relacionadas con la adopción, tales como: emisiones e infraestructura. Adicionalmente, pocos estudios han profundizado en las relaciones de infraestructura de transporte, emisiones y seguridad alimentaria [11], [46].

Figura 7. Número de artículos publicados en las categorías de adopción, capacidad tecnológica (baterías) y comportamiento del consumidor adoptados por los autores

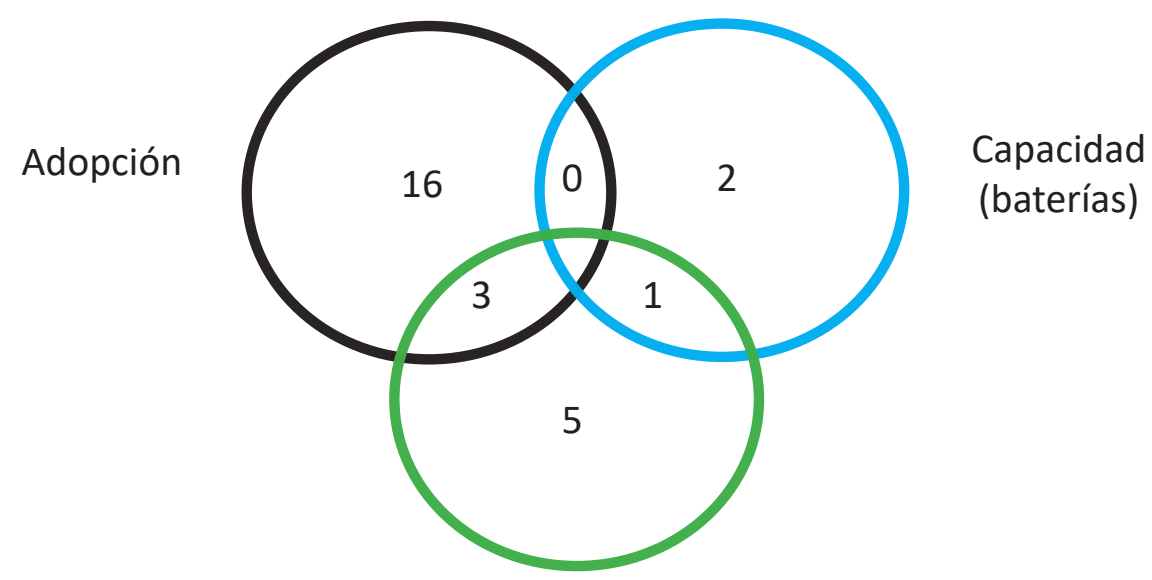

Comportamiento del consumidor

Fuente: elaboración propia. 
Otro aspecto de análisis desarrollado está relacionado con el factor de impacto de las publicaciones y este a su vez con el número de citaciones obtenidas de los documentos en cada categoría. Las figuras 8 y 9 presentan el número de citaciones obtenidas en las categorías de análisis de adopción, emisiones, infraestructura, comportamiento del consumidor y capacidad tecnológica. Se identifica que las categorías de análisis con mayor impacto están relacionadas con la reducción de emisiones atmosféricas, en segundo lugar, las emisiones e infraestructura y en tercer lugar el comportamiento del consumidor de la tecnología vehicular eléctrica. Finalmente, de las categorías emergentes se identifican la infraestructura y la capacidad tecnológica, esta última con mayor número de publicaciones desde otros enfoques metodológicos [15], [39].

Figura 8. Número de citaciones en las categorías de adopción, emisiones e infraestructura

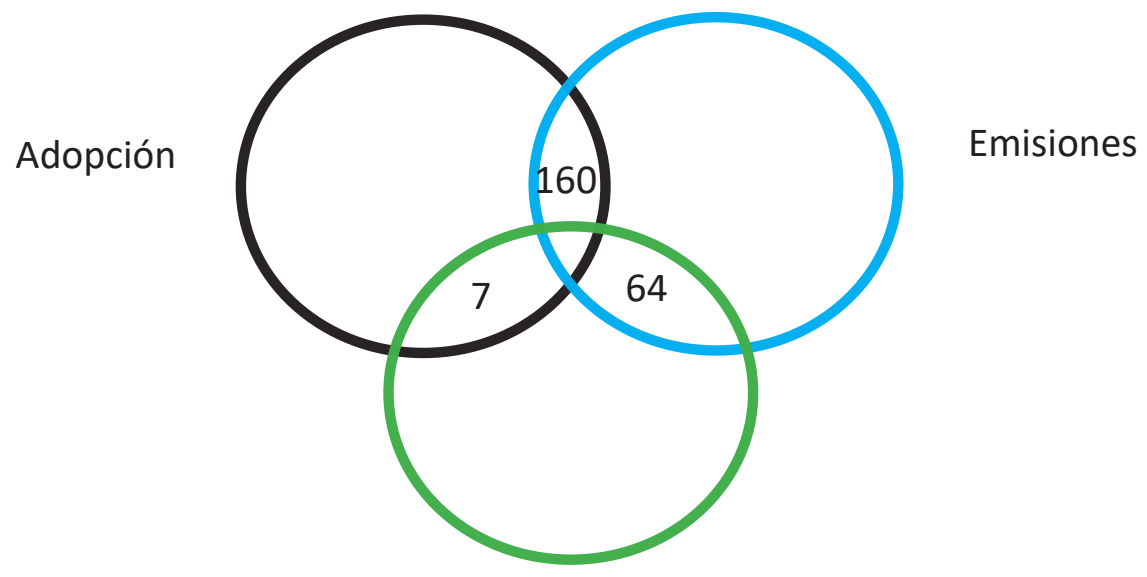

Infraestructura

Fuente: elaboración propia.

Figura 9. Número de citaciones en las categorías de adopción, capacidad tecnológica (baterías) y comportamiento del consumidor

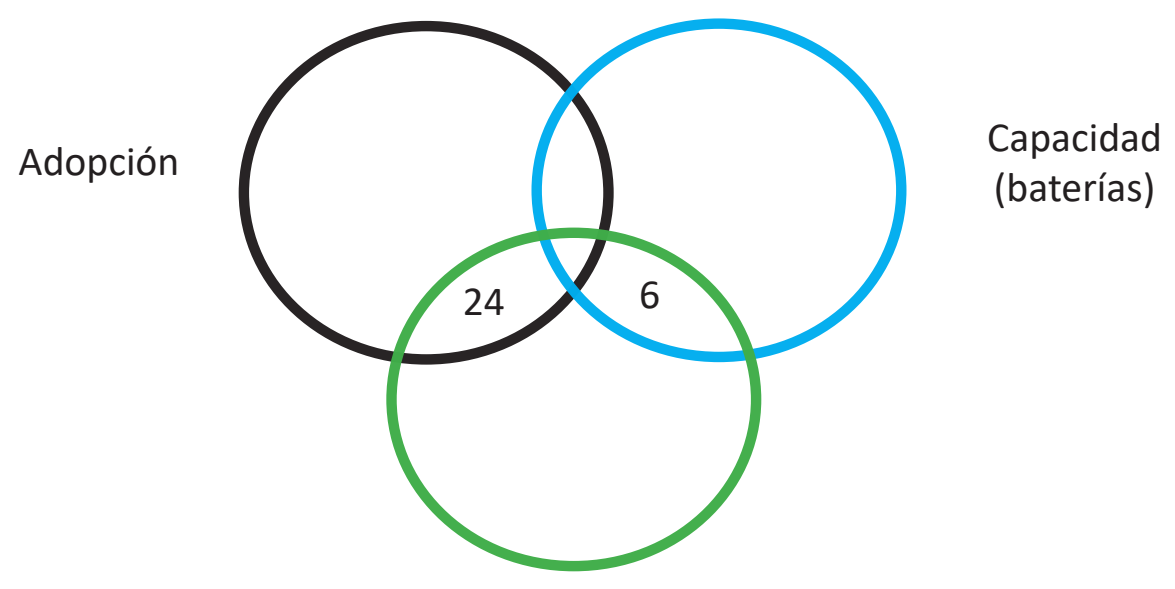

Comportamiento del consumidor

Fuente: elaboración propia. 
En resumen, las publicaciones identificadas que han utilizado la metodología de simulación de dinámica de sistemas en las diferentes categorías de análisis propuestas en este artículo permiten identificar la conjunción de algunos temas que han sido abordados desde una intervención sistémica. A partir de escenarios de simulación y de la evaluación de políticas, que la dinámica de sistemas posibilita realizar, los estudios analizados muestran la conjunción de diferentes categorías que contribuyen en el diseño e implementación de política para la transición de tecnologías limpias.

Una intervención sistémica contribuye a entender las dinámicas del comportamiento y permite fortalecer el diseño de política de transición tecnológica [21], [47]. Por lo tanto, la dinámica de sistemas se convierte en una herramienta útil en la evaluación y análisis de escenarios para entender la conjunción de problemas que podrían afectar el sistema de transporte de un país o región [48].

\section{CONCLUSIONES}

El artículo contribuyó con una visión que presenta la dinámica de las publicaciones en las cuales la simulación a través de la dinámica de sistemas permite identificar diferentes categorías de análisis y la conjunción que en ellas se pueden encontrar. Si bien un incremento en el número de artículos publicados ha volcado su interés en los impactos y la mitigación de las emisiones atmosféricas producidas por los vehículos de combustión interna, existen categorías de análisis emergentes tales como infraestructura y capacidad tecnológica relacionadas con el comportamiento del consumidor que pueden contribuir a la transición de vehículos eléctricos. Algunas de las contribuciones que pueden identificarse en el artículo son las siguientes.

Primero, la transición de vehículos eléctricos de carga o pasajeros requiere de intervenciones sistémicas que permitan entender los efectos causales de diferentes categorías de análisis, ejemplo infraestructura, emisiones, capacidades y comportamiento de consumidor frente a la adquisición de nuevas tecnologías. Estudios previos muestran la necesidad de realizar este tipo de evaluaciones que contribuyen en el desarrollo sostenible y sostenibilidad de los sistemas de transporte [49].

Segundo, categorías de análisis emergentes poco tratadas en la literatura son identificadas. Estas categorías pueden ser combinadas para obtener mejores conocimientos de la dinámica de los sistemas de transporte, lo cual podría contribuir a la sostenibilidad de los sistemas de transporte [50].

Tercero, se requiere de nuevas estrategias complementarias a la simulación que permitan adoptar políticas y diseños que suavicen los procesos de transición tecnológica en el caso de vehículos eléctricos [51].

\section{AGRADECIMIENTOS}

Los autores quieren agradecer el soporte financiero de la Universidad Militar Nueva Granada en el marco del proyecto de iniciación científica titulado "Modelado de alternativas sostenibles de transporte y sus implicaciones en la emisión de gases de efecto invernadero" con código PIC-ECO-2872. Los autores también les gustaría agradecer por las sugerencias y comentarios de los pares evaluadores.

\section{REFERENCIAS}

[1] M. Espinosa, Á. I. Cadena, y E. Behrentz, "Challenges in greenhouse gas mitigation in developing countries: A case study of the Colombian transport sector", Energy Policy, vol. 124, pp. 111-122, 2019.

[2] L. M. Benvenutti, M. Uriona-Maldonado, y L. M. S. Campos, "The impact of $\mathrm{CO} 2$ mitigation policies on light vehicle fleet in Brazil", Energy Policy, vol. 126, pp. 370-379, 2019.

[3] J. E. Martínez-Jaramillo, S. Arango-Aramburo, K. C. Álvarez-Uribe, y P. Jaramillo-Álvarez, "Assessing the impacts of transport policies through energy system simulation: The case of the Medellin Metropolitan Area, Colombia", Energy Policy, vol. 101, pp. 101-108, 2017.

[4] N. Ito, K. Takeuchi, y S. Managi, "Willingness-to-pay for infrastructure investments for alternative fuel vehicles", Transp. Res. Part $D$ Transp. Environ., vol. 18, n. ${ }^{\circ}$ 1, pp. 1-8, 2013.

[5] S. Y. Park, J. W. Kim, y D. H. Lee, "Development of a market penetration forecasting model for Hydrogen Fuel Cell Vehicles considering infrastructure and cost reduction effects", Energy Policy, vol. 39, n. ${ }^{\circ}$ 6, pp. 3307-3315, 2011.

[6] A. Muñoz-Villamizar, J. R. Montoya-Torres, y J. Faulin, "Impact of the use of electric vehicles in collaborative urban transport 
networks: A case study", Transp. Res. Part D Transp. Environ., vol. 50, pp. 40-54, 2017.

[7] S. P. Shepherd, "A review of system dynamics models applied in transportation", Transp. BTransport Dyn., vol. 2, n 2, pp. 83-105, 2014.

[8] J. D. Sterman, Business dynamics: Systems Thinking and Modeling for a Complex World. Boston: McGraw-Hill, 2000.

[9] M. M. Herrera, J. Rosero-García, y O. Casas, "Systemic analysis of the adoption of electric vehicle technologies in Colombia", Int. Rev. Mech. Eng., vol. 11, n. ${ }^{\circ}$ 4, 2017.

[10] D. Ospina, S. Zapata, M. Castañeda, I. Dyner, A. J. Aristizábal, y N. Escalante, "Model for evaluating $\mathrm{CO} 2$ emissions and the projection of the transport sector", Int. J. Electr. Comput. Eng., vol. 8, n. ${ }^{\circ}$ 3, pp. 1781-1787, 2018.

[11] J. Orjuela, M. M. Herrera, y W. Casilimas, "Impact analysis of transport capacity and food safety in Bogota", en 2015 Workshop Engineering Application, Bogotá, 2015, pp. 7-13.

[12] J. D. Sterman, Business Dynamics: Systems thinking nd Modelling for a Complez World. Boston: McGraw-Hill, 2000.

[13] J. W. Forrester, Industrial dynamics. Cambridge, Massachusetts: M.I.T. Press, 1961.

[14] H. Rahmandad, N. Repenning, y J. Sterman, "Effects of feedback delays on learning", Syst. Dyn. Rev., vol. 25, n. ${ }^{\circ} 4$, pp. 309-338, 2009.

[15] M. J. Eppstein, D. K. Grover, J. S. Marshall, y D. M. Rizzo, "An agent-based model to study market penetration of plug-in hybrid electric vehicles", Energy Policy, vol. 39, n. ${ }^{\circ}$ 6, pp. 3789-3802, 2011.

[16] Y. Geum, S. Lee, y Y. Park, "Combining technology roadmap and system dynamics simulation to support scenario-planning: A case of car-sharing service", Comput. Ind. Eng., vol. 71, pp. 37-49, 2014.

[17] D. Wheat, “What Can System Dynamics Learn From the Public Policy Implementation Literature?", Syst. Res. Behav. Sci., vol. 27, n. ${ }^{\circ}$, pp. 425-442, 2010.

[18] M. Jimenez, C. J. Franco, y I. Dyner, "Diffusion of renewable energy technologies: The need for policy in Colombia", Energy, vol. 111, pp. 818-829, 2016.

[19] M. M. Herrera, I. Dyner, y F. Cosenz, "Assessing the effect of transmission constraints on wind power expansion in northeast Brazil”, Util. Policy, vol. 59, ag. 2019.

[20] M. M. Herrera, F. Cosenz, y I. Dyner, "How to support energy policy coordination? Findings from the Brazilian wind industry", Electr. J., vol. 32, n. ${ }^{\circ} 8,2019$.

[21] J. Struben y J. D. Sterman, "Transition challenges for alternative fuel vehicle and transportation systems", Environ. Plan. B Plan. Des., vol. 35, n. ${ }^{\circ}$ 6, pp. 1070-1097, 2008.

[22] E. Bivona y G. Montemaggiore, "Understanding short-and long-term implications of 'myopic' flet maintenance policies: a system dynamics application to a city bus company", Syst. Dyn. Rev., vol. 26, n. ${ }^{\circ}$ 3, pp. 195-215, 2010.

[23] W. B. Fontoura, G. de L. D. Chaves, y G. M. Ribeiro, "The Brazilian urban mobility policy: The impact in São Paulo transport system using system dynamics", Transp. Policy, vol. 73, pp. 51-61, 2019.

[24] S. Fallah-Fini, H. Rahmandad, K. Triatis, y J. de la Garza, "Optimizing highway maintanance operations: dynamic considerations", Syst. Dyn. Rev., vol. 26, n. 3, pp. 216-238, 2010.

[25] P. Pfaffenbichler, G. Emberger, y S. Shepherd, "A system dynamics approach to land use transport interation modelling: the strategic model MARS and its application", Syst. Dyn. Rev., vol. 26, n. ${ }^{\circ} 3$, pp. 262-282, 2010.

[26] L. Cox et al., "Exploring synergies between transit investment and dense redevelopment: A scenario analysis in a rapidly urbanizing landscape", Landsc. Urban Plan., vol. 167, pp. 429-440, 2017.

[27] H. Vafa-Arani, S. Jahani, H. Dashti, J. Heydari, y S. Moazen, "A system dynamics modeling for urban air pollution: A case study of Tehran, Iran", Transp. Res. Part D Transp. Environ., vol. 31, pp. 21-36, 2014.

[28] Z. Wang, Y. Zhang, L. Lian, y C. Chu, “Evaluating transportation infrastructure investment on a regional level: a system dynamics simulation", Simulation, vol. 94, n. ${ }^{\circ} 10$, pp. 943-954, 2018.

[29] H. Wang, K. Zhang, J. Chen, Z. Wang, G. Li, y Y. Yang, "System dynamics model of taxi management in metropolises: Economic and environmental implications for Beijing", J. Environ. Manage., vol. 213, pp. 555-565, 2018.

[30] L. Wen y L. Bai, "System Dynamics Modeling and Policy Simulation for Urban Traffic: 
a Case Study in Beijing", Environ. Model. Assess., vol. 22, n.o 4, pp. 363-378, 2017.

[31] P. Liu, C. Liu, J. Du, y D. Mu, “A system dynamics model for emissions projection of hinterland transportation", J. Clean. Prod., vol. 218, pp. 591-600, 2019.

[32] T. Ercan, N. C. Onat, y O. Tatari, "Investigating carbon footprint reduction potential of public transportation in United States: A system dynamics approach", J. Clean. Prod., vol. 133, pp. 1260-1276, 2016.

[33] J. Sim, "The influence of new carbon emission abatement goals on the truck-freight transportation sector in South Korea", J. Clean. Prod., vol. 164, n' 2017, pp. 153-162, 2017.

[34] L. M. M. Benvenutti, A. B. Ribeiro, y M. Uriona, "Long term diffusion dynamics of alternative fuel vehicles in Brazil", J. Clean. Prod., vol. 164, pp. 1571-1585, 2017.

[35] G. Noto, “Urban Transportation Governance and Wicked Problems: A system and performance oriented approach", tesis Ph. D., Departamento de Ciencia Política y Relaciones Internacionales (DEMS), Universidad de Palermo, Palermo, 2015.

[36] G. Egilmez y O. Tatari, "A dynamic modeling approach to highway sustainability: Strategies to reduce overall impact", Transp. Res. Part A Policy Pract., vol. 46, n. ${ }^{\circ}$ 7, pp. 1086-1096, 2012.

[37] N. C. Onat, M. Kucukvar, O. Tatari, y G. Egilmez, "Integration of system dynamics approach toward deepening and broadening the life cycle sustainability assessment framework: a case for electric vehicles", Int. J. Life Cycle Assess., vol. 21, n. ${ }^{\circ}$ 7, pp. 10091034, 2016.

[38] X. Zhang, Y. Liang, y W. Liu, "Pricing model for the charging of electric vehicles based on system dynamics in Beijing", Energy, vol. 119, pp. 218-234, 2017.

[39] R. Hein, P. R. Kleindorfer, y S. Spinler, "Valuation of electric vehicle batteries in vehicle-to-grid and battery-to-grid systems", Technol. Forecast. Soc. Change, vol. 79, n. ${ }^{\circ}$, pp. 1654-1671, 2012.

[40] M. Braz da Silva y F. Moura, "Electric vehicle diffusion in the Portuguese automobile market”, Int. J. Sustain. Transp., vol. 10, $\mathrm{n}^{\circ} 2$, pp. 49-64, 2016.
[41] M. M. Herrera, J. Rosero, y O. Casas, "Systemic Analysis of the Adoption of Electric Vehicle Technologies in Colombia", Int. Rev. Mech. Eng., vol. 11, pp. 256-269, abr. 2017.

[42] K. Kieckhäfer, T. Volling, y T. S. Spengler, “A hybrid simulation approach for estimating the market share evolution of electric vehicles", Transp. Sci., vol. 48, n. ${ }^{\circ}$ 4, pp. 651-670, 2014.

[43] Y. Lee, C. Kim, y J. Shin, “A hybrid electric vehicle market penetration model to identify the best policy mix: A consumer ownership cycle approach", Appl. Energy, vol. 184, n. ${ }^{\circ}$ 2016, pp. 438-449, 2016.

[44] X. Zhou, R. Zhao, L. Cheng, y X. Min, “Impact of policy incentives on electric vehicles development: a system dynamics-based evolutionary game theoretical analysis", Clean Technol. Environ. Policy, vol. 21, n. ${ }^{\circ}$ 5, pp. 1039-1053, 2019.

[45] K. Laurischkat y D. Jandt, "Techno-economic analysis of sustainable mobility and energy solutions consisting of electric vehicles, photovoltaic systems and battery storages", J. Clean. Prod., vol. 179, pp. 642-661, 2018.

[46] J. A. Orjuela-Castro, M. M. Herrera-Ramírez, y W. Adarme-Jaimes, "Warehousing and transportation logistics of mango in Colombia: A system dynamics model", Rev. Fac. Ing., vol. 26, n. ${ }^{\circ}$ 44, pp. 71-84, 2017.

[47] M. Uriona y S. S. Grobbelaar, "Innovation system policy analysis through system dynamics modelling: A systematic review", Sci. Public Policy, vol. 46, n. ${ }^{\circ} 1$, pp. 28-44, 2019.

[48] D. Fiorello, F. Fermi, y D. Bielanska, "The ASTRA model for strategic assessment of transport policies”, Syst. Dyn. Rev., vol. 26, n. ${ }^{\circ}$, pp. 283-290, 2010.

[49] J. Dong, Y. Xu, B. gang Hwang, R. Ren, y Z. Chen, "The impact of underground logistics system on urban sustainable development: A system dynamics approach", Sustain., vol. 11, n.o 5, 2019.

[50] Y. Shi, T. Arthanari, X. Liu, y B. Yang, "Sustainable transportation management: Integrated modeling and support", J. Clean. Prod., vol. 212, pp. 1381-1395, 2019.

[51] J.-H. Lewe, L. F. Hivin, y D. N. Mavris, “A multi-paradigm approach to system dynamics modeling of intercity transportation", Transp. Res. Part E Logist. Transp. Rev., vol. 71, pp. 188-202, 2014. 


\section{APÉNDICE I}

\begin{tabular}{|c|c|c|c|c|c|c|c|}
\hline Citas & Autores & Titulo & Año & Revista & DOI & ISSN & $\begin{array}{l}\text { Citación } \\
\text { por año }\end{array}$ \\
\hline 89 & S. Shepherd & $\begin{array}{l}\text { Factors affecting future } \\
\text { demand for electric } \\
\text { vehicles: A model based } \\
\text { study }\end{array}$ & 2012 & $\begin{array}{c}\text { Transport } \\
\text { Policy }\end{array}$ & 10.1016/j.tranpol.2011.12.006 & 0967070X & 12,71 \\
\hline 46 & G. Egilmez & $\begin{array}{c}\text { A dynamic modeling } \\
\text { approach to highway } \\
\text { sustainability: Strategies } \\
\text { to reduce overall impact }\end{array}$ & 2012 & $\begin{array}{l}\text { Transportation } \\
\text { Research } \\
\text { Part A }\end{array}$ & 10.1016/j.tra.2012.04.011 & $0965-8564$ & \\
\hline 38 & N. Onat & $\begin{array}{l}\text { Integration of system } \\
\text { dynamics approach } \\
\text { toward deepening and } \\
\text { broadening the life cycle } \\
\text { sustainability assessment } \\
\text { framework: a case for } \\
\text { electric vehicles }\end{array}$ & 2016 & $\begin{array}{l}\text { International } \\
\text { Journal of } \\
\text { Life Cycle } \\
\text { Assessment }\end{array}$ & $10.1007 / \mathrm{s} 11367-016-1070-4$ & 9483349 & 12,67 \\
\hline 29 & R. Hein & $\begin{array}{l}\text { Valuation of electric } \\
\text { vehicle batteries in } \\
\text { vehicle-to-grid and } \\
\text { battery-to-grid systems }\end{array}$ & 2012 & $\begin{array}{l}\text { Technological } \\
\text { Forecasting } \\
\text { and Social } \\
\text { Change }\end{array}$ & 10.1016/j.techfore.2012.06.002 & 401625 & 4,14 \\
\hline 20 & X. Zhang & $\begin{array}{l}\text { Pricing model for the } \\
\text { charging of electric } \\
\text { vehicles based on system } \\
\text { dynamics in Beijing }\end{array}$ & 2017 & Energy & 10.1016/j.energy.2016.12.057 & 3605442 & 10,00 \\
\hline 18 & G. Harrison & $\begin{array}{l}\text { An exploratory policy } \\
\text { analysis of electric } \\
\text { vehicle sales competition } \\
\text { and sensitivity to } \\
\text { infrastructure in Europe }\end{array}$ & 2017 & $\begin{array}{l}\text { Technological } \\
\text { Forecasting } \\
\text { and Social } \\
\text { Change }\end{array}$ & 10.1016/j.techfore.2016.08.007 & 401625 & 9,00 \\
\hline 17 & $\begin{array}{c}\text { K. } \\
\text { Kieckhäfer }\end{array}$ & $\begin{array}{l}\text { Analyzing manufacturers' } \\
\text { impact on green } \\
\text { products' market } \\
\text { diffusion - the case of } \\
\text { electric vehicles }\end{array}$ & 2017 & $\begin{array}{l}\text { Journal of } \\
\text { Cleaner } \\
\text { Production }\end{array}$ & 10.1016/j.jclepro.2016.05.021 & 9596526 & 8,50 \\
\hline 15 & $\begin{array}{c}\text { K. } \\
\text { Kieckhäfer }\end{array}$ & $\begin{array}{c}\text { A hybrid simulation } \\
\text { approach for estimating } \\
\text { the market share } \\
\text { evolution of electric } \\
\text { vehicles }\end{array}$ & 2014 & $\begin{array}{l}\text { Transportation } \\
\text { Science }\end{array}$ & $10.1287 /$ trsc. 2014.0526 & 411655 & 3,00 \\
\hline 14 & C. Lu & $\begin{array}{l}\text { A key stakeholder- } \\
\text { based financial subsidy } \\
\text { stimulation for Chinese } \\
\text { EV industrialization: } \\
\text { A system dynamics } \\
\text { simulation }\end{array}$ & 2017 & $\begin{array}{l}\text { Technological } \\
\text { Forecasting } \\
\text { and Social } \\
\text { Change }\end{array}$ & 10.1016/j.techfore.2017.01.022 & $0040-1625$ & \\
\hline 12 & $\begin{array}{l}\text { M. Braz da } \\
\text { Silva }\end{array}$ & $\begin{array}{l}\text { Electric vehicle diffusion } \\
\text { in the Portuguese } \\
\text { automobile market }\end{array}$ & 2016 & $\begin{array}{l}\text { International } \\
\text { Journal of } \\
\text { Sustainable } \\
\text { Transportation }\end{array}$ & $10.1080 / 15568318.2013 .853851$ & 15568318 & 4,00 \\
\hline
\end{tabular}

Fuente: elaboración propia. 